\title{
Dense instanton-dyon liquid model: Diagrammatics
}

\author{
Yizhuang Liu, ${ }^{*}$ Edward Shuryak, ${ }^{\dagger}$ and Ismail Zahed ${ }^{*}$ \\ Department of Physics and Astronomy, Stony Brook University, Stony Brook, New York 11794-3800, USA
}

(Received 11 April 2018; published 19 July 2018)

\begin{abstract}
We revisit the instanton-dyon liquid model in the confined phase by using a nonlinear Debye-Huckel (DH) resummation for the Coulomb interactions induced by the moduli, followed by a cluster expansion. The organization is shown to rapidly converge and yields center symmetry at high density. The dependence of these results on a finite vacuum angle is also discussed. We also formulate the hypernetted chain (HNC) resummation for the dense instanton-dyon liquid and use it to estimate the liquid pair correlation functions in the DH limit. At very low temperature, the dense limit interpolates between chains and rings of instanton-anti-instanton dyons and a bcc crystal, with strong topological and magnetic correlations.
\end{abstract}

DOI: 10.1103/PhysRevD.98.014023

\section{INTRODUCTION}

This work is a continuation of our earlier studies [1] of the gauge topology in the confining phase of a theory with the simplest gauge group $S U(2)$. We suggested that the confining phase below the transition temperature is an "instanton dyon" (and antidyon) plasma which is dense enough to generate strong screening. The dense plasma is amenable to standard mean-field methods.

The basic ingredients of the instanton-dyon liquid model are Kraan-van-Baal-Lee-Lu (kvBLL) instantons with finite holonomies [2]. Diakonov and Petrov [3,4] have argued that the KvBLL instantons split into instanton dyons in the confined phase below the critical temperature, and recombine above it in the deconfined phase. These observations have also been checked numerically [5]. The dissociation of instantons into constituents was advocated originally by Zhitnitsky and others [6], and more recently by Unsal and collaborators [7] using controlled semiclassical approximations. When light quarks are added, center symmetry and chiral symmetry are found to be tied $[1,8-10]$.

To put the present investigation into perspective, we recall that detailed lattice simulations using cooling techniques have shown that the dominant gauge configurations to the light hadronic correlators stem from correlated instantons and anti-instantons [11], in agreement with most

\footnotetext{
*yizhuang.liu@stonybrook.edu edward.shuryak@stonybrook.edu

*ismail.zahed@stonybrook.edu
}

Published by the American Physical Society under the terms of the Creative Commons Attribution 4.0 International license. Further distribution of this work must maintain attribution to the author(s) and the published article's title, journal citation, and DOI. Funded by SCOAP. predictions from the instanton liquid model [12] (and references therein). Yet the instanton liquid model does not confine. So what changes are needed in this model to achieve confinement? Are these changes substantial enough to modify the established successes of this model?

More than a decade ago, it was noted that since the deconfinement transition through the Polyakov line requires a nonzero holonomy, all semiclassical configurations including instantons and anti-instantons need to be modified. The answer was the KvBLL instanton which was found to interpolate between a pair of instanton dyons at low temperature and an instanton at high temperature. The instanton dyons carry Abelian electric and magnetic charges, and in the confined phase form a plasmalike state. This description accounts for the correct gluino condensate in supersymmetric Yang-Mills theory [13] and qualitatively explains the confinement-deconfinement transition in pure Yang-Mills theory [4]. Recently, we have carried several studies of instanton-dyon ensembles using mean-field approximations without and with light quarks in the confined phase for a center-symmetric phase with holonomy fixed at $\frac{1}{2}$ [1].

The purpose of this paper is to revisit the instanton-dyon liquid model without quarks, at low temperature in the confined or center-symmetric phase, by improving on the mean-field approximation through the use of many-body resummations pertinent to dense liquids. This resummation will allow us to probe higher densities or lower temperatures which will bring us closer to a description of the vacuum state for Yang-Mills. In particular, we will show that the resummation

(1) Provides a specific interpolation between bionlike correlations in the dilute phase, and mostly screened interactions in the dense phase. The bionlike correlations are in support of the semiclassical approximations used in [7], while the screened correlations are in support of the mean-field analysis [1]; 
(2) Allows for the derivation of the free energy for arbitrary holonomy with a maximum for the $\frac{1}{2}$-holonomy in support of the arguments in [1];

(3) Yields a specific dependence of the free energy on the vacuum angle, that indicates a decrease of the critical temperature with vacuum angle. For a small vacuum angle it is in agreement with current lattice estimates [14];

(4) Suggests the possibility of a (disordered) crystal in the very dense regime. By Poisson duality, the crystal can be viewed either as an arrangement of topological charges (instantons) or magnetic charges (monopoles), implying a dual role for the instantons and monopoles in the description of the vacuum state.

In Sec. II we briefly review the salient aspects of the instanton-dyon liquid model. We perform a nonlinear Debye-Huckel resummation of the Coulomb interactions stemming from the moduli space, and combine them with a cluster expansion of the Coulomb interactions originating from the streamlines. We show that the expansion is rapidly converging and the phase center is symmetric already in the second cluster approximation. In Sec. III we also show how the multichain and rings can be further resummed beyond the leading clusters and make them explicit with some applications. In Sec. IV, we extend our arguments to a finite vacuum angle $\theta$. In Sec. V, we discuss a larger class of resummation pertinent for dense systems referred to as a hypernetted chain (HNC) resummation. In Sec. VI, we suggest that a melted crystal of instanton dyons and antiinstanton dyons may provide a semiclassical description of a Yang-Mills ensemble at very low temperature. Our conclusions are in Sec. VII. In the Appendix we outline the elements for a future molecular dynamics simulation.

\section{THERMAL YANG-MILLS}

The chief aspects of the instanton-dyon liquid model have been discussed in $[1,3,4]$ to which we refer the reader for more details. Here, we briefly recall the key elements which will be useful in setting up the statistical Coulomb analysis using many-body techniques. For two-colors the KvBLL instanton (anti-instanton) splits into $L, M(\bar{L}, \bar{M})$ instanton dyons for large holonomies. $M$ carries $(+,+)$ and $L$ carries $(-,-)$ for (electric-magnetic) charges, with fractional topological charges $\nu$ and $\bar{\nu}=1-\nu$. The holonomy is fixed by the large x-asymptotics $\lim _{x \rightarrow \infty}\left\langle A_{4}^{3}\right\rangle=$ $2 \pi T \nu \tau^{3} / 2$ at fixed temperature $T$. In the confined phase with $\nu=\frac{1}{2}$ and moderate gauge coupling $\alpha_{s} \leq 1$ the instanton-dyon actions $S_{L}=2 \pi \nu / \alpha_{s}$ and $S_{M}=2 \pi \bar{\nu} / \alpha_{s}$ are still large, justifying their use in a semiclassical description of the thermal Yang-Mills phase. Throughout, the instanton and anti-instanton dyons will carry a finite core size which we will specify below.

A semiclassical ensemble of instanton-anti-instanton dyons can be regarded as a statistical ensemble of semiclassical charges interacting mostly through their moduli space for like instanton or anti-instanton dyons and through streamlines for unlike instanton-anti-instanton dyons. The grand partition function for such an ensemble is of the form (zero vacuum angle)

$$
\begin{aligned}
\mathcal{Z}[T, f] \equiv & \sum_{[K]} \prod_{i_{L}=1}^{K_{L}} \prod_{i_{M}=1}^{K_{M}} \prod_{i_{\bar{L}}=1}^{K_{\bar{L}}} \prod_{i_{\bar{M}}=1}^{K_{\bar{M}}} \\
& \times \int \frac{f d^{3} x_{L i_{L}}}{K_{L} !} \frac{f d^{3} x_{M i_{M}}}{K_{M} !} \frac{f d^{3} y_{\bar{L} i_{\bar{L}}}}{K_{\bar{L}} !} \frac{f d^{3} y_{\bar{M} i_{\bar{M}}}}{K_{\bar{M}} !} \\
& \times e^{-V(x-y)+\ln \operatorname{det}(G[x] G[y])} .
\end{aligned}
$$

The streamline interactions $V$ are large and of order $1 / \alpha_{s}$. They are attractive between like $D \bar{D}$ and repulsive between unlike $D \bar{D}$ [15]. Their relevant form for our considerations will be detailed below. In contrast, the moduli induced interactions captured in the $\left(K_{L}+K_{M}\right)^{2}$ matrix $G[x]$ and in the $\left(K_{\bar{L}}+K_{\bar{M}}\right)^{2}$ matrix $G[y]$ are of order $\alpha_{s}^{0}$. While the explicit form of these matrices can be found in $[3,4]$, it is sufficient to note here that these induced interactions are attractive between unlike instanton dyons and repulsive between like instanton dyons. The bare fugacity $f$ will be regarded as an external parameter in what follows. Note that in the absence of $V, \mathcal{Z} \rightarrow \mathcal{Z}_{D} \mathcal{Z}_{\bar{D}}$ where each factor can be exactly rewritten in terms of a three-dimensional effective theory.

\section{A. Effective action}

The streamline interaction part $V$ can be bosonized using the complex fields $b \pm i \sigma$ through standard tricks. Here $b, \sigma$ refers to the Abelian magnetic and electric potentials stemming from the instanton-dyon charges. Also, each moduli determinant in (1) can be fermionized using ghost fields, and the ensuing Coulomb factors bosonized using complex $w, \bar{w}$ fields also through standard tricks as detailed in $[3,4]$. The net result of these repeated fermionizationbosonization procedures is an exact three-dimensional effective action ( $\mathrm{p}$-space)

$$
\begin{aligned}
-S_{B}[b, \sigma, w, \bar{w}]= & \int d^{3} p\left[\frac{1}{4}(b-i \sigma) V^{-1}(p)(b+i \sigma)\right. \\
& +4 \pi\left(\nu f e^{w}+\bar{\nu} f e^{-w}\right) \\
& \left.+4 \pi\left(\nu f e^{\bar{w}}+\bar{\nu} f e^{-\bar{w}}\right)\right]
\end{aligned}
$$

subject to the constraint from the moduli (x-space)

$$
\begin{aligned}
& -\frac{T}{4 \pi} \nabla^{2}(w)+4 \pi f \sinh (w)=\frac{T}{4 \pi} \nabla^{2}(b-i \sigma) \\
& -\frac{T}{4 \pi} \nabla^{2}(\bar{w})+4 \pi f \sinh (\bar{w})=\frac{T}{4 \pi} \nabla^{2}(b+i \sigma) .
\end{aligned}
$$


Equations (2) and (3) allow us to rewrite exactly the partition function (1) in terms of a three-dimensional effective theory. In [1] we have analyzed this partition function using the Debye-Huckel (one-loop) approximation. Here we will seek a more systematic organization of the dense phase described by (2) and (3) that is more appropriate for the description of the confined phase at low temperature.

\section{B. Cluster expansion}

Our starting point is the linearization of (3) around $w=0$ which amounts to the solution

$$
w(p)=\frac{p^{2}}{p^{2}+M^{2}}(b-i \sigma)(p)
$$

with the squared screening mass $M^{2}=\frac{16 \pi f}{T}$. Inserting (4) into (2), we can carry the cluster expansion for the $4 \pi f$ terms by integrating over the $b, \sigma$ fields as the measure is Gaussian in the partition function defined now in terms of the three-dimensional effective action (2). The result at second order is

$$
\begin{aligned}
\frac{\ln Z}{V_{3}}= & 8 \pi f+(4 \pi f)^{2}\left(\nu^{2}+\bar{\nu}^{2}\right) \int d^{3} r\left(e^{-V_{1}(r)}-1\right) \\
& +(4 \pi f)^{2}(2 \nu \bar{\nu}) \int d^{3} r\left(e^{-V_{2}(r)}-1\right)
\end{aligned}
$$

with

$$
V_{1}(p)=-V_{2}(p)=-\frac{p^{4} V(p)}{\left(p^{2}+M^{2}\right)^{2}} .
$$

While the instanton-anti-instanton dyon interaction is accessible numerically, for simplicity we will use here only its Coulomb asymptotic form $V(p) \approx \frac{4 \pi C_{D}}{\alpha_{s} p^{2}}$ with $C_{D}=2$, so that

$$
V_{1}(r)=-V_{2}(r) \approx \frac{M C_{D}}{2 \alpha_{s}}\left(-\frac{2}{M r}+1\right) e^{-M r} .
$$

The large r-interaction between the pairs with magnetic charge $0(\bar{M} M$ and $\bar{L} L)$ turns repulsive at large r, while that between the pairs with magnetic charge $2(\bar{M} L$ and $\bar{L} M)$ turns attractive. Remarkably, the sign of the induced interaction between the pairs in (7) is flipped in comparison to the unscreened or bare interaction between the pairs, a sign of overscreening.

The chief effect of the moduli constraint in (3) and (4) is to induce a nonlinear Debye-Huckel screening effect between the charged instanton and anti-instanton dyons through the Mayer functions $e^{-V_{1,2}}-1$. This is a rearrangement of the many-body dynamics that does not assume diluteness. In contrast, the cluster expansion in (5)

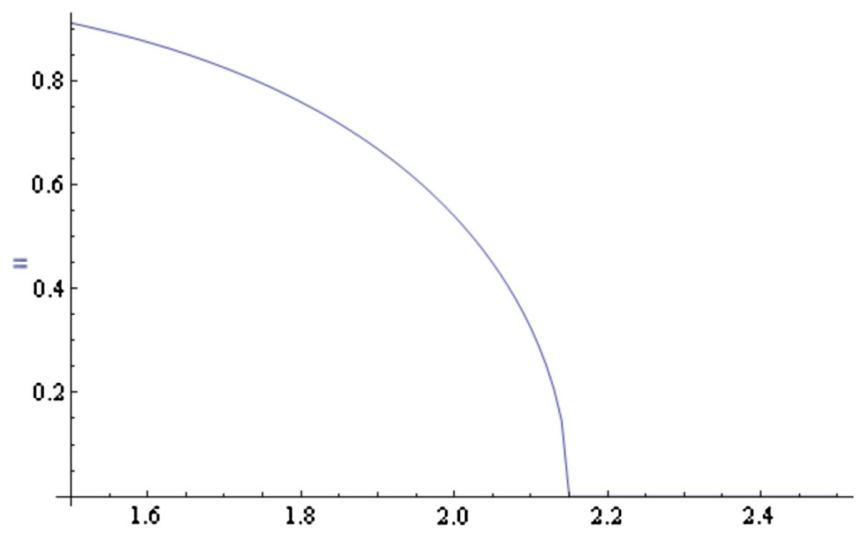

FIG. 1. Polyakov line $\mathrm{P}=|\cos (\pi \nu)|$ versus $\mathrm{m}$.

is limited to the second cumulant and subsume diluteness in the ensemble of $D, \bar{D}$ but with nonlinear DebyeHuckel effective interactions. This shortcoming will be addressed later.

For small $r$, we need to set a core for the attractive pair with magnetic charge 2 . We choose the core to be $a=\frac{1}{T}$. As a result (5) plus the perturbative contribution reads

$$
\begin{aligned}
z_{b}(m, \nu) & =\frac{\ln Z}{V_{3} T^{3}}-\frac{4 \pi^{2}}{3} \nu^{2} \bar{\nu}^{2} \\
& =\frac{m^{2}}{2}+F(m, \nu)-\frac{4 \pi^{2}}{3} \nu^{2} \bar{\nu}^{2}
\end{aligned}
$$

with $m=\frac{M}{T}$ and $V(x)=(-2 / x+1) e^{-x}$ and

$$
\begin{gathered}
F(m, \nu)=\frac{\pi m}{4}\left(\nu^{2}+\bar{\nu}^{2}\right) \int_{1} x^{2}\left(e^{-\frac{m C_{D}}{2 \alpha_{s}} V(x)}-1\right) \\
+\frac{\pi m}{2} \nu \bar{\nu} \int_{c 1} x^{2}\left(e^{\frac{m C_{D}}{2 \alpha_{s}} V(x)}-1\right) .
\end{gathered}
$$

For $C_{D} \approx 2$ and $\alpha_{s}=1$, the transition from a centersymmetric (confining) to a center-asymmetric (deconfining) phase occurs for $m_{c} \approx 2.1,2.3$ for the two choices of the cutoff parameter $c 1=1,0$. The choice $c 1=0$ corresponds to the formal argument presented in [7]. In terms of the density of charged particles $n=8 \pi f$, the transition occurs for $n \approx 2 T^{3}$. For large density, the screening length scales like $\sqrt{T} / \sqrt{n}$, while the average separation scales like $1 / n^{\frac{1}{3}}$. Our expansion is therefore justified. In Fig. 1 we show the behavior of the Polyakov line versus $m$ for the cutoff choice $c 1=1$.

\section{OPEN AND CLOSED CHAINS}

To go beyond the second cumulant approximation in (3) with bare fugacities, we will discuss in this section a systematic way for resumming all tree diagrams between the charged particles, and also all ring diagrams with an arbitrary number of trees at the charged vertices. One of the 
chief effects of the resummation of all the trees is a redefinition of the fugacities of the charged particles as we will show below.

\section{A. Diagrammatics}

A systematic book-keeping procedure for the resummation of all the trees and the rings with redefined fugacities follows from a semiclassical treatment of the Coulomb-like field theory

$$
\mathcal{L}=-\frac{1}{2} \phi^{T} \mathcal{V}^{-1} \phi+f_{1}\left(e^{i \phi_{1}}+e^{i \phi_{3}}\right)+f_{2}\left(e^{i \phi_{2}}+e^{i \phi_{4}}\right)
$$

with $f_{1}=4 \pi f \nu, f_{2}=4 \pi f \bar{\nu}$, the effective fields in three dimensions $\phi=\left(\phi_{1}, \phi_{2}, \phi_{3}, \phi_{4}\right)^{T}$,

$$
\mathcal{V}=\left(\begin{array}{ll}
0 & \mathbf{V} \\
\mathbf{V} & 0
\end{array}\right) \quad \mathbf{V}=\left(\begin{array}{ll}
F_{1} & F_{2} \\
F_{2} & F_{1}
\end{array}\right)
$$

and the Mayer functions $-F_{1,2}=e^{-\beta V_{1,2}}-1$. The $4 \times 4$ block structure follows from the fact that the statistical ensemble consists of four species of charged particles $D=L, M$ (filled circle) and $\bar{D}=\bar{L}, \bar{M}$ (open circle). The block off-diagonal character of $\mathcal{V}$ follows from the fact that the Mayer functions $-F_{1,2}$ resum the nonlinear Debye screening induced by the moduli between like instanton dyons and are left acting only between unlike $D \bar{D}$ instanton-anti-instanton dyons. It can be checked that (10) reproduces all Coulomb diagrams with the correct symmetry and weight factors as Feynman graphs when the vertices are linked by single lines only as illustrated in Figs. 2 and 3.

A resummation of all trees and rings with arbitrary trees at the vertices amounts to a one-loop expansion around the saddle point approximation to (10) which is given by

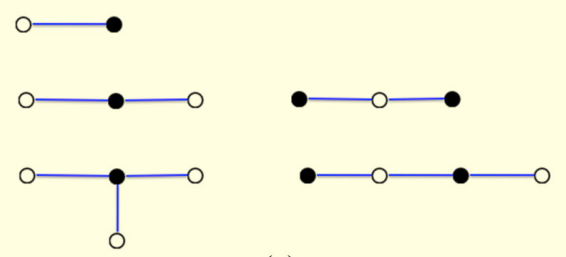

(a)
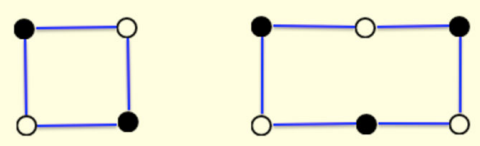

(b)

FIG. 2. Typical open chain contributions to $\Omega_{\text {tree }}$ (a) and closed chain or ring contributions to $\Omega_{\text {ring }}$ (b). The filled circle refers to $D=L, M$ and open circle refers to $\bar{D}=\bar{L}, \bar{M}$.
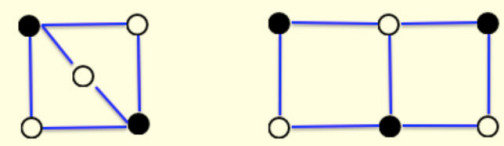

FIG. 3. Examples of two-loop contributions not included in the fugacity redefined one-loop or ring resummation.

$$
\phi_{c}^{T}=i \mathcal{V}(p=0)\left(f_{1} e^{i \phi_{1 c}}, f_{2} e^{i \phi_{2 c}}, f_{1} e^{i \phi_{3 c}}, e^{i \phi_{4 c}}\right) .
$$

Because of symmetry, the solution satisfies $\phi_{1}=\phi_{3}$, $\phi_{2}=\phi_{4}$. If we define $\alpha_{1}=i \phi_{1 c}, \alpha_{2}=i \phi_{2 c}$ and use the symmetry, then (12) reads

$$
\begin{aligned}
& \alpha_{1}=c_{1} f_{1} e^{\alpha_{1}}+c_{2} f_{2} e^{\alpha_{2}} \\
& \alpha_{2}=c_{1} f_{2} e^{\alpha_{2}}+c_{2} f_{1} e^{\alpha_{1}} .
\end{aligned}
$$

Here

$$
c_{1,2}=\int d^{3} x\left(e^{-\beta V_{1,2}}-1\right)
$$

are the integrated Mayer functions. The saddle point contribution which resums all connected trees yields the pressure

$$
\Omega_{\text {tree }}=\frac{\ln Z_{\text {tree }}}{V_{3}}=f_{1} e^{\alpha_{1}}\left(2-\alpha_{1}\right)+f_{2} e^{\alpha_{2}}\left(2-\alpha_{2}\right)
$$

with $\alpha_{1,2}$ solutions to the nonlinear classical equations (13). The resummed rings with arbitrary trees follow by expanding (12) around the classical solution (13) to one loop. The result is

$$
\Omega_{\text {ring }}=-\frac{1}{2} \int \frac{d^{3} p}{(2 \pi)^{3}}(\ln (1+\mathbb{A})-\mathbb{A})
$$

with in p-space

$\mathbb{A}=-\left(\tilde{f}_{1}^{2}+\tilde{f}_{2}^{2}\right) F_{1}^{2}+2 \tilde{f}_{1} \tilde{f}_{2} F_{2}^{2}+\tilde{f}_{1}^{2} \tilde{f}_{2}^{2}\left(F_{1}^{2}-F_{2}^{2}\right)^{2}$.

In the special case with $F \equiv F_{1} \approx-F_{2}$, the one-loop result simplifies to

$$
\mathbb{A}=-\left(\tilde{f}_{1}+\tilde{f}_{2}\right)^{2} F^{2}
$$

where $\tilde{f}_{1,2}=f_{1,2} e^{\alpha_{1,2}}$ are the tree-modified fugacities.

\section{B. Approximations}

The preceding expansion around the small fugacities follows by seeking the classical solution to (13) in powers $f_{1,2}$ or $\alpha_{1} \approx c_{1} f_{1}+c_{2} f_{2}, \alpha_{2} \approx c_{1} f_{2}+c_{2} f_{1}$. The tree contributions to the pressure in (15) to quadratic order are 


$$
\Omega_{\text {tree }} \approx 2\left(f_{1}+f_{2}\right)+c_{1}\left(f_{1}^{2}+f_{2}^{2}\right)+2 c_{2} f_{1} f_{2}
$$

in agreement with (5). For large fugacities $f_{1,2}$ and for $c_{1}=-c_{2}=-c<0$, the solution to (16) satisfies $\alpha_{1}=$ $-\alpha_{2}=\alpha$ with $\nu e^{\alpha}=\bar{\nu} e^{-\alpha}=\sqrt{\bar{\nu} \nu}$. As a result, the leading contribution in (5) is now changed to

$$
8 \pi f \rightarrow 8 \pi \tilde{f} \equiv 8 \pi f \sqrt{4 \bar{\nu} \nu} .
$$

The resummation of all the trees for large bare fugacities amounts to dressing the bare fugacities through $f \rightarrow \tilde{f}$ in a cluster expansion for the rings with no trees attached as illustrated in Fig. 2(b). Some of the diagrams not included in the dressed fugacity expansion with ring diagrams are illustrated in Fig. 3 which are of the two-loop types. The first appear in the fifth cumulant, and the second in the sixth cumulant. So this reorganization resums a large class of diagrams. The resumation is exact up to the fifth cumulant, but approximate beyond. Remarkably, in the center-symmetric phase with $\nu=\bar{\nu}=\frac{1}{2}$, (20) amounts to the fugacity of noninteracting instanton and anti-instanton dyons, as all Coulomb interactions from the (linearized) moduli and the streamlines average out.

In general, the solution to (13) for intermediate fugacities is not amenable analytically. One way to go beyond the second cumulant approximation (19) at low density is to insert the leading solutions $\alpha_{1} \approx c_{1} f_{1}+c_{2} f_{2}, \alpha_{2} \approx c_{1} f_{2}+$ $c_{2} f_{1}$ in (15) without expanding the exponent,

$\Omega_{\text {tree }} \approx 4 \pi f\left(\frac{1}{2}+b\right)(2 K b+2) e^{-2 K b}+(b \rightarrow-b)$

where we have set $\nu=\frac{1}{2}+b, K=4 \pi f c$, and noted that $c_{1}=-c_{2}=-c<0$. Equation (21) resums all tree contributions with charge vertices that include an arbitrary number of two-body links. Equation (19) follows by expanding the exponents to first order in $f$. We note that (21) always has a maximum at $b=0$ or $\nu=\frac{1}{2}$ for positive $c$ which is center symmetric (confining). This conclusion remains unchanged when the ring contributions are added. Indeed, we note that the ring contribution (16) is an increasing function of the combination $\tilde{f}_{1}+\tilde{f}_{2}$ or more specifically

$\tilde{f}_{1}+\tilde{f}_{2} \approx 8 \pi f\left(\frac{1}{2}+b\right) e^{-2 K b}+8 \pi f\left(\frac{1}{2}-b\right) e^{-2 K b}$

with

$$
2 K=8 \pi f c=\frac{2 \pi}{m} \int d x x^{2}\left(e^{\frac{m C_{D}}{2 \alpha_{S}} V(x)}-1\right)
$$

using the previous notations. For $2 K>4$ or $c f>\frac{1}{2 \pi}$, this combination has a maximum away from 0 and competes against the classical contribution towards the center-symmetric solution. For $m<10$ we have $2 K<4$. The ring contribution preserves center symmetry.

The center-symmetric phase can be probed more accurately by setting $\nu=\frac{1}{2}-b$. The semiclassical equation (22) reads

$$
\alpha=-K\left(\frac{1}{2}+b\right) e^{\alpha}+K\left(\frac{1}{2}-b\right) e^{-\alpha} .
$$

At $b=0$ we have $\alpha=0$. We now can solve (24) by expanding exactly around $b=0$. Since $\alpha$ is an odd function of $b$, we seek a solution to (24) using $\alpha=x_{1} b+x_{2} b^{3}+$ $\cdots$, with $x_{1}$ satisfying

$$
x_{1}=-2 K-K x_{1} .
$$

Since the leading contribution to the pressure is given by

$\frac{\ln Z}{V_{3}} \approx 4 \pi f \nu e^{\alpha}\left(1-\frac{\alpha}{2}\right)+4 \pi f \bar{\nu} e^{-\alpha}\left(1+\frac{\alpha}{2}\right)+$ c.c.

its expanded form to order $\mathcal{O}\left(b^{4}\right)$ reads

$$
\begin{aligned}
\frac{\ln Z}{V_{3}} & \approx 8 \pi f-8 \pi f \frac{2 K}{K+1} b^{2}+\mathcal{O}\left(b^{4}\right) \\
& \rightarrow 8 \pi f\left(\frac{1+K \sqrt{4 \nu \bar{\nu}}}{1+K}\right)
\end{aligned}
$$

where the last relation follows after restoring the full $\nu$ dependence. Equation (27) shows that only the open chains with no treelike-star insertions contribute to the leading $b^{2}$ and therefore $\sqrt{\nu \bar{\nu}}$ in the pressure. Note that (27) is independent of the integrated Mayer function $c$ in $K=4 \pi f c$ in the center-symmetric phase and/or large fugacities, in agreement with (20).

\section{FINITE VACUUM ANGLE $\theta$}

At finite vacuum angle $\theta$, the bare fugacities for $\phi_{1,2}$ are now complex and given by $f_{1}=4 \pi f \nu e^{\frac{i \theta}{2}}$ and $f_{2}=4 \pi f \bar{\nu} e^{\frac{i \theta}{2}}$, while the bare fugacities for $\phi_{3,4}$ are their conjugate $f_{1,2}^{\dagger}$. For $c_{1}=c_{2}=-c<0$, we first note that the solution to the analogue of the classical equations (13) at finite $\theta$ satisfies $\alpha_{3,4}=\alpha_{1,2}^{\dagger}$, and $\alpha_{1}=-\alpha_{2}=\alpha$, with $\alpha$ complex and satisfying

$$
\alpha=-K e^{-i \frac{\theta}{2}} \bar{\nu} e^{\alpha^{\dagger}}+K e^{-i \frac{\theta}{2}} \nu e^{-\alpha^{\dagger}} .
$$

The solution for small or large fugacities can be obtained analytically. We now discuss them sequentially.

\section{A. Large $K$}

For large fugacities or large $K$, the solution to (28) in leading order gives $e^{\alpha}=\sqrt{\nu / \bar{\nu}}$ independently of $K$. In this 
limit, the summation of all the tree diagrams amounts to a dressed fugacity with a leading (dimensionless) pressure

$$
\frac{\ln Z}{V_{3} T^{3}} \rightarrow \frac{m^{2}}{2} \sqrt{4 \nu \bar{\nu}} \cos (\theta / 2)-\frac{4 \pi^{2}}{3} \nu^{2} \bar{\nu}^{2}
$$

with $m^{2}=\frac{2 n}{T^{3}}$ and including the perturbative contribution. Equation (29) resums all the tree cumulant contributions at finite $\theta$ and is to be compared to (8) and (9) with only the second cumulant retained. Equation (29) implies a transition from the center-symmetric (confined) phase to the center-asymmetric (deconfined) phase at a critical temperature

$$
\frac{T_{c}(\theta)}{T_{c}(0)}=\left(\cos \left(\frac{\theta+2 k \pi}{N_{c}}\right)\right)^{\frac{1}{3}}
$$

with $T_{c}^{3}(0)=\frac{12 n}{\pi^{2}}$ for $N_{c}=2$. Although our derivation was for $N_{c}=2$, our arguments for the resummation of the trees extend to any $N_{c}$. Also, (29) and (30) were derived for $|\theta|<\pi$ in a $2 \pi$-branch with $k=0$. The general result is multibranch and $2 \pi$-periodic following the substitution $\theta \rightarrow \theta+2 k \pi$. Numerical lattice simulations have established that the transition temperature $T_{c}(\theta)$ decreases with $\theta$ as $(k=0$ branch $)$

$$
\frac{T_{c}(\theta)}{T_{c}(0)}=1-R_{\theta} \theta^{2}+\mathcal{O}\left(\theta^{2}\right)
$$

with $R_{\theta}=0.0175(7)$ for $N_{c}=3$ [14], in good agreement with $R_{\theta}=1 / 6 N_{c}^{2}=0.0185$ from (30). Our result (30) is predictive of the $N_{c}$ dependence of $R_{\theta}$ and of the higher $\theta$ coefficients, with a cusp at $T_{c}(\pi) / T_{c}(0)=1 / 2^{3}$ at the $C P$ symmetric point. This point is actually a tricritical point where the $C P$ breaking first order transition line at $\theta=\pi$ meets the first order transition cusp from (30). Although (30) suggests that the $C P$ transition line reduces to a point for $N_{c}=2$, this conclusion requires further amendments as it occurs at 0 temperature where the liquid is very dense, requiring additional resummations, some of which will be detailed below.

\section{B. Intermediate $K$}

The onset of the center-symmetric phase depends on the details of the arrangement of the parameters $K, \theta$, as (29) was only established for large $K$ or high density. The center-symmetric phase can be probed more accurately for different densities or $K$ by again setting $\nu=\frac{1}{2}-b$ in (28), and solving exactly around $b=0$. The result for the pressure to order $\mathcal{O}\left(b^{4}\right)$ is $\frac{\ln Z}{V_{3}}=8 \pi f \cos \frac{\theta}{2}-8 \pi f 2 K \frac{K \cos \frac{\theta}{2}-1}{K^{2}-1} b^{2}+\mathcal{O}\left(b^{4}\right)$

which is seen to reduce to (27) at $\theta=0$. At finite vacuum angle $\theta$, the expanded result (32) develops a singularity at $K=4 \pi f c=1$, the origin of which requires a more careful analysis.

In general, we have $\alpha_{1}=-\alpha_{2}$ and $\alpha_{3}=-\alpha_{4}$. At finite $\theta$, all $\alpha_{1,2,3,4}$ are complex and satisfy the coupled equations

$$
\begin{aligned}
& \alpha_{1}=-K e^{-i \frac{\theta}{2}} \nu e^{\alpha_{3}}+K e^{-i \frac{\theta}{2} \bar{\nu}} e^{-\alpha_{3}} \\
& \alpha_{3}=-K e^{+i \frac{\theta}{2}} \nu e^{\alpha_{1}}+K e^{+i \frac{\theta}{2} \bar{\nu}} e^{-\alpha_{1}}
\end{aligned}
$$

At small $\theta$, these equations can be analyzed numerically by analytically continuing $\theta \rightarrow-i \theta$, so that

$$
\begin{aligned}
& \alpha_{1}=-K e^{-\frac{\theta}{2}}\left(\frac{1}{2}+b\right) e^{\alpha_{3}}+K e^{-\frac{\theta}{2}}\left(\frac{1}{2}-b\right) e^{-\alpha_{3}} \\
& \alpha_{3}=-K e^{+\frac{\theta}{2}}\left(\frac{1}{2}+b\right) e^{\alpha_{1}}+K e^{+\frac{\theta}{2}}\left(\frac{1}{2}-b\right) e^{-\alpha_{1}},
\end{aligned}
$$

with $\alpha_{1,2,3,4}$ now all real. If we define

$f(b, K, \theta, x)=-K e^{-\frac{\theta}{2}}\left(\frac{1}{2}+b\right) e^{x}+K e^{-\frac{\theta}{2}}\left(\frac{1}{2}-b\right) e^{-x}$

then $\alpha_{3}=x$ satisfies the transcendental equation

$$
f(b, K,-\theta, f(b, K, \theta, x))-x=0 .
$$

A numerical analysis of (36) reveals a solution with a three-branch structure in the parameter space. In the region $b \ll 1$ around the center-symmetric state, it turns out that for $K$ sufficiently close to 1 but less than 1 there exists a critical $b_{c}(K, \theta)$. For $b<b_{c}(K, \theta)$, the expansion leading to (32) is valid. However for $b>b_{c}(K, \theta)$, the branch which leads to (32) no longer exists, and the solution to (36) jumps to a third branch. For $K \geq 1$ and small $b$ only the third branch exists and will lead to the expansion (32) for $K>1$. For $K=1$, the solution is more tricky. In Fig. 4 we show the solution $x(b)$ at $\theta=0.1$ and $K=1$. In terms of the pressure, it is interesting to see if a "window" appears for $K=1$. For imaginary $\theta$, we can see a window for $(1 / \cosh (\theta / 2))<K<1$ numerically. Indeed, for $\theta=0.01$ and $K=0.99999$ we show in Fig. 5 the pressure $\frac{\ln Z}{V_{3}}$ versus $b$, with no maximum at $b=0$. In contrast, for $K$ outside the window, we always have $b=0$ as the maximum, which corresponds to the center-symmetric phase. The window disappears for $\theta=0$. Its occurrence at finite $\theta$ signals the incompleteness of the tree resummation for $K$ in the range $(1 / \cos (\theta / 2))<K<1$ after analytical continuation. 

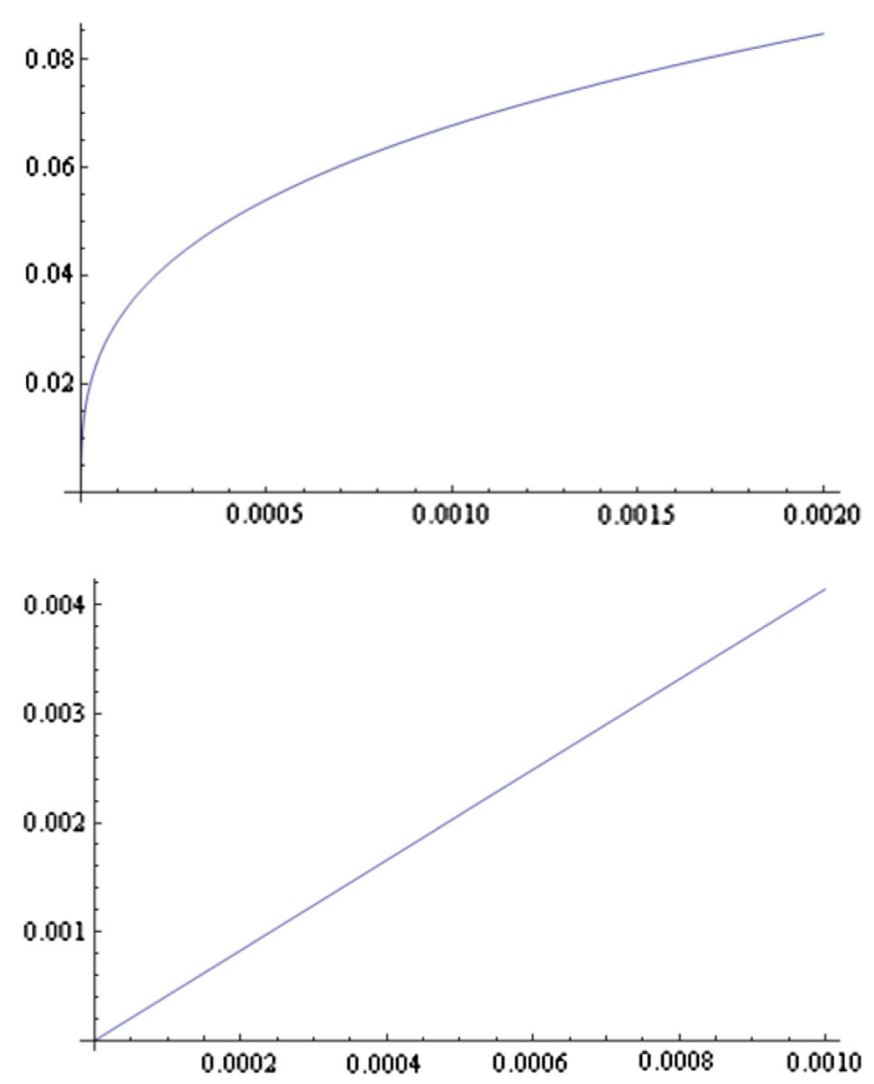

FIG. 4. $x(b)$ as a function of $b$ for $K=1, \theta=0.1$ (upper) and $K=1.01, \theta=0.1$ (lower).

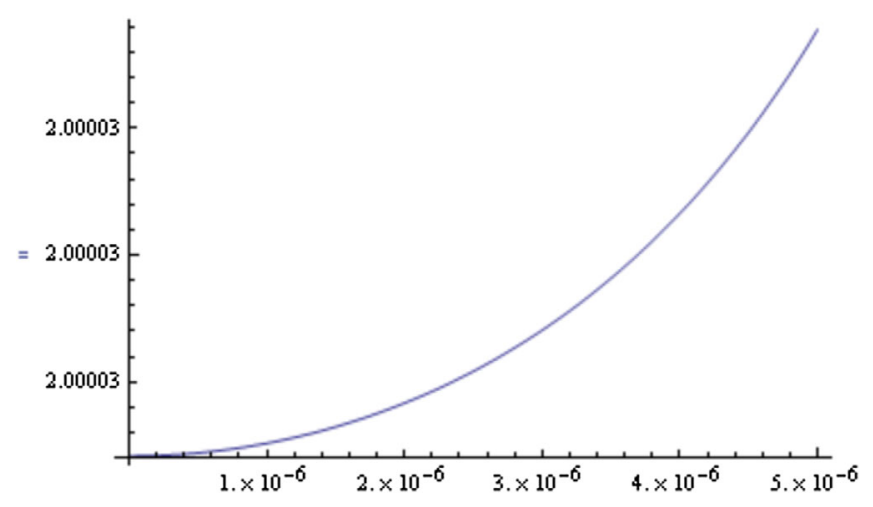

FIG. 5. Pressure $\frac{\ln Z}{V_{3}}$ versus $b$ for $\theta=0.01$ and $K=0.99999$.

\section{HYPERNETTED CHAINS}

The static properties of a strongly coupled fluid are usually expressed in terms of few-body reduced distribution functions of which the two-body distribution $g\left(\vec{r}_{1}, \vec{r}_{2}\right)$ or radial distribution $g\left(r_{12}\right)$ is the standard example. The radial distribution function describes how the fluid density varies as a function of distance from a reference particle, providing a link between the microscopic content of the fluid and its macroscopic structure. $g\left(r_{12}\right)$ can be obtained either from simulations using

molecular dynamics (see below) or by solving the Ornstein-Zernicke (OZ) equation [16] subject to an additional closure relation. In this section we discuss such a closure in the form of the well-known hypernetted chain resummation adapted to our dense dyon liquid. For that, we will provide a diagrammatic derivation based on our effective field theory (10).

\section{A. Diagrammatic derivation}

In the dense instanton-dyon liquid, the radial distribution following from the many-body analysis of (10) is a $4 \times 4$ matrix with instanton-dyon entries $g^{i j}\left(r_{12}\right)$. It is related to the irreducible density two-point correlation function through

$$
h^{i j}(r)=g^{i j}(r)-1 \equiv e^{-\beta \mathcal{V}^{i j}(r)+\chi^{i j}(r)}-1
$$

where the use of the barometric form in (37) defines $\chi^{i j}(r)$, and the $4 \times 4$ matrix $\mathcal{V}$ is given in (11). $\chi^{i j}$ obeys a set of formal matrix equations

$$
\begin{aligned}
\chi^{i j} & =\chi_{a}^{i j}+\chi_{b}^{i j} \\
\chi_{a}^{i j} & =c^{i l} \rho^{l} c^{l j}+c^{i l} \rho^{l} c^{l m} \rho^{m} c^{m j}+\cdots \\
c^{i j} & =h^{i j}-\chi_{a}^{i j}
\end{aligned}
$$

where $\rho^{i j}=\rho^{i} \delta^{i j}$ is a diagonal matrix with species density $\rho^{i}$. We now provide a diagrammatic derivation of (38) using the effective formulation (10).

The total pair correlation function $h^{i j}$ follows from summing all irreducible graphs with two external vertices fixed between $\overrightarrow{0}$ and $\vec{r}$. Between these two vertices we can hang an arbitrary number of independent two-point functions as illustrated in Fig. 6(a). The minimal insertion that cannot be decomposed into such a hanging structure is denoted by $-\beta \mathcal{V}+\chi$ with $\beta=\frac{1}{T}$. The diagrams contributing to $\chi$ can be separated into type (a) and type (b). Type (a) have at least one cutting point, i.e., a vertex that one can cut to split the diagram into two disconnected pieces as illustrated in Fig. 6(b), while type (b) have none as illustrated in Fig. 6(c). For type (a), we can further count by enumerating the number of cutting points and define a summation over all possible two-point diagrams that can be put between two nearest cutting points as $c(r)$, which defines the direct correlation function. It is readily seen that $c=h-\chi_{a}$. With these definitions in mind, simple diagrammatic arguments yield (38). The HNC approximation amounts to setting $\chi_{b}=0$. In this case, (38) can be cast in the more standard form

$$
\begin{aligned}
& h^{i j}=c^{i j}+c^{i k} \rho^{k} \star h^{k j} \\
& c^{i j}=-\beta \mathcal{V}^{i j}+h^{i j}-\ln \left(1+h^{i j}\right)
\end{aligned}
$$

where $\star$ means convolution in $\mathrm{x}$-space. The first of these equations is known as the Ornstein-Zernicke equation, 


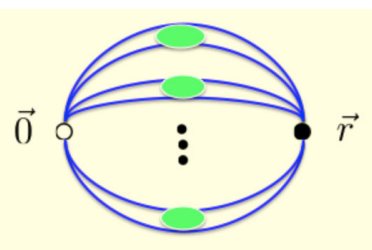

(a)

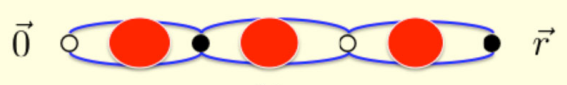

(b)

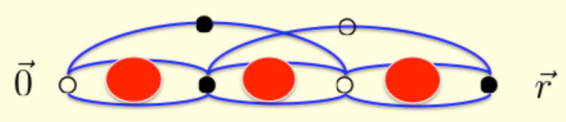

(c)

FIG. 6. (a) Typical diagrammatic contribution to the pair correlation function $h(r)$ where each hanging ring is $-\beta \mathcal{V}+\chi$. (b) Typical contribution to $\chi_{a}$. (c) Typical contribution to $\chi_{b}$.

while the second equation as the HNC closure condition. The interaction energy per three-volume and therefore the pressure can be reconstructed using the pair correlation function, for instance

$$
\frac{\mathcal{E}}{V_{3}}=\left(\frac{2 N}{V_{3}}\right) \frac{1}{2} \sum_{i, j} \int d^{\beta} r \beta \mathcal{V}^{i j}(r) h^{i j}(r) .
$$

\section{B. Linear and nonlinear DH approximations}

The linear Debye-Huckel (DH) approximation follows by performing one iteration in the $\mathrm{OZ}$ equation with the initial condition $h=0$ or $c \approx-\beta \mathcal{V}$, to obtain formally in $\mathrm{p}$-space

$$
h_{\mathrm{DH}}=\frac{-\beta \mathcal{V}}{1+\beta \rho \mathcal{V}} .
$$

For the instanton-dyon ensemble we have $\rho=\rho_{1}=\rho_{2}=$ $M^{2} T / 8$ and $V_{1}=-V_{2}=-V(p)=-\frac{8 \pi p^{2}}{\left(p^{2}+M^{2}\right)^{2}}$ in $\mathcal{V}$, so that

$h_{\mathrm{DH}}=\frac{\beta V}{1-(2 \beta \rho V)^{2}}\left(\begin{array}{cc}2 \beta \rho V & 1 \\ 1 & 2 \beta \rho V\end{array}\right) \otimes\left(1-\sigma_{1}\right)$.

Here $\sigma_{1}$ is a Pauli matrix. Equation (42) defines two independent pair correlation functions in $\mathrm{p}$-space

$$
\begin{aligned}
& h_{M M}=h_{L L}=-h_{M L}=\frac{2 \rho(8 \pi \beta)^{2} p^{4}}{\left(p^{2}+M^{2}\right)^{4}-(16 \pi \beta \rho)^{2} p^{4}} \\
& h_{M \bar{M}}=h_{L \bar{L}}=-h_{M \bar{L}}=\frac{(8 \pi \beta) p^{2}\left(p^{2}+M^{2}\right)^{2}}{\left(p^{2}+M^{2}\right)^{4}-(16 \pi \beta \rho)^{2} p^{4}} .
\end{aligned}
$$

For $\rho=M^{2} T / 8$ the denominator

$$
\left(p^{2}+M^{2}\right)^{4}-4 \pi^{2} p^{4} M^{4}
$$

is negative for $p>\frac{M}{\sqrt{2 \pi-1}} \approx \frac{M}{2}$. The spatial cutoff $a=\frac{1}{T}$ used earlier translates to a p-cutoff of $T$. Since $M \approx 2 T$, the negative range is physically not relevant. These observations are similar to the ones encountered in the $\mathrm{DH}$ analysis of the electric and magnetic correlation functions in [1] (first reference).

The HNC equations (39) allow us to go beyond the DH approximation in the dense ensemble, but require a numerical calculation. Here, we only mention that a simple nonlinear correction to the $\mathrm{DH}$ result follows from (39) by retaining the leading correction to the direct correlation function, namely $c^{i j} \approx-\beta \mathcal{V}^{i j}+\frac{1}{2}\left(h^{i j}\right)^{2}$, and we use it to iterate the OZ equation after the substitution $h \rightarrow h_{\mathrm{DH}}$. The net effect is a nonlinear correction to the $\mathrm{DH}$ result (41) in p-space:

$$
h_{\mathrm{DH} 2}=\frac{-\beta \mathcal{V}+\frac{1}{2} h_{\mathrm{DH}}^{2}}{1+\rho\left(\beta \mathcal{V}-\frac{1}{2} h_{\mathrm{DH}}^{2}\right)} .
$$

\section{INSTANTON-DYON CRYSTAL}

At even higher fugacity or density, the instanton and antiinstanton dyons are expected to crystallize. A typical bcc cubic crystal arrangement with low energy is illustrated in Fig. 7. Recall that the resummed $M \bar{M}$ interactions and $L \bar{L}$ interactions are repulsive, while the $L M$ and $\bar{L} M$ interactions are attractive. In the bcc crystal structure, we note that the nearest neighbor $L M$ vertices are close to an instanton configuration, while their alternate nearest neighbors $\bar{L} M$ vertices are close to a magnetically charged twobion. We will refer to this as crystal duality. We note that holographic dyonic crystals composed only of $L, M$ in saltlike or popcornlike crystal configurations were suggested in [17] for a holographic description of dense matter.

The instanton and anti-instanton dyons considered throughout are the lightest of a Kaluza-Klein tower with higher winding numbers which carry larger actions (more massive). We expect them to crystallize following a similar pattern, albeit with higher windings. We expect this tower of three-dimensional crystal arrangements along the extra winding direction to be dual to a four-dimensional crystal arrangement of monopoles and antimonopoles (or instantons and anti-instantons by crystal duality), using the Poisson duality suggested in [7]. Remarkably, the resulting four-dimensional and semiclassical description at very low temperature can be either described as instantonlike (topologically charged) or monopolelike (magnetically charged) as the two descriptions are tied by crystal duality.

The crystal is an idealized description of the strongly coupled and dense phase as both the low temperature and the quantum fluctuations cause it to melt. The melted form of Fig. 7 resembles an ionic liquid with four species of ions 
with strong local order. This semiclassical description of the Yang-Mills state at very low temperature appears to reconcile the instanton liquid model without confinement, with the 't Hooft-Mandelstam proposal with confinement. In the former, the low temperature thermal state is composed of a liquid of instantons and anti-instantons, while in the latter it is a superfluid of monopoles and antimonopoles with bions as precursors [7]. The dual descriptions allow for a center-symmetric thermal state with both strong and local topological and magnetic correlations.

\section{A. Crystal energy}

To assess the crystal contribution to the pressure at high density, we first evaluate the interaction energy for the crystal structure in Fig. 7. Consider the $L$ instanton dyon sitting in the center of the $\mathrm{M}$ cell. The interaction summation within the L-lattice reads

$$
2 E_{L}=\sum_{n_{1}, n_{2}, n_{3} \neq 0}\left(1-(-1)^{n_{1}+n_{2}+n_{3}}\right) V\left(\vec{r}_{n_{1}, n_{2}, n_{3}}\right) .
$$

The mutual interaction between the L- and M-lattice is

$$
-2 E_{M L}=\sum_{n_{1}, n_{2}, n_{3}}\left(1-(-1)^{n_{1}+n_{2}+n_{3}}\right) V\left(\frac{1}{2} \vec{r}_{111}+\vec{r}_{n_{1}, n_{2}, n_{3}}\right) .
$$

In momentum space, these sums can be cast using the dual lattice $\vec{b}_{n}=\frac{2 \pi}{a} \vec{n}$, using the identity

$$
\sum_{a_{n}} e^{i p \cdot a_{n}}=\sum_{n} \delta\left(p-b_{n}\right)
$$

The results are

$$
\begin{aligned}
2 E_{L} & =\sum_{n}\left(V\left(b_{n}\right)-V\left(b_{n}+\frac{\pi}{a}(1,1,1)\right)\right) \\
-2 E_{M L} & =\sum_{n}(-1)^{n}\left(V\left(b_{n}\right)-V\left(b_{n}+\frac{\pi}{a}(1,1,1)\right)\right)
\end{aligned}
$$

where we made use of

$$
e^{i b_{n} \cdot \frac{r_{111}}{2}}=(-1)^{n_{1}+n_{2}+n_{3}}=(-1)^{n} .
$$

Both the x-space sums (46) and (47) and the p-space sums (49) can only be carried numerically. However, we note that the $\mathrm{x}$-sum is converging exponentially and can be approximated by the leading contribution involving only the nearest neighbors,

$$
E_{L} \equiv M E_{L}(\tilde{a}) \approx M\left(6 V(\tilde{a})-4 V\left(\frac{\sqrt{3} \tilde{a}}{2}\right)\right)
$$

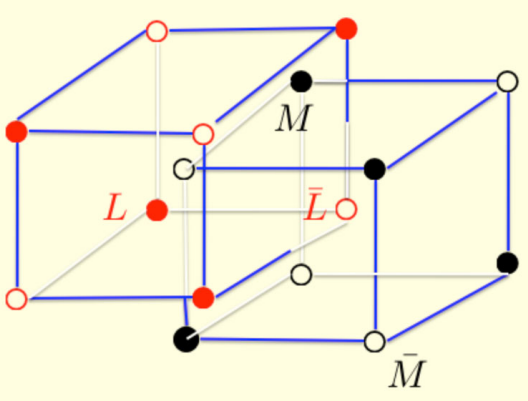

FIG. 7. Three-dimensional bcc crystal composed of the instanton and antinstanton dyons with the lowest winding, for two colors.

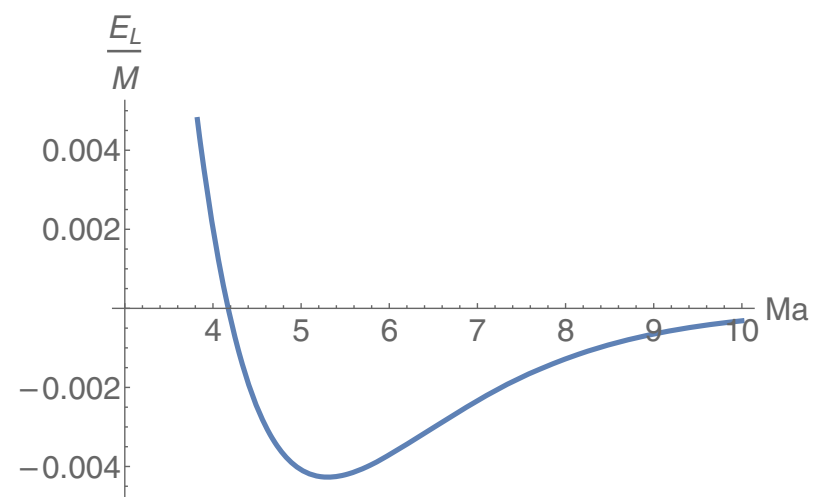

FIG. 8. Crystal energy for the bcc arrangement $E_{L} / M$ versus $M a$ as given in (51) with $\alpha_{s}=1$.

with $V(x)=\frac{1}{\alpha_{s}}\left(-\frac{2}{x}+1\right) e^{-x}$ from (7) with $C_{D}=2$. Here we have set $\tilde{a}=M a$, with $M^{2}=\frac{2 n}{T}$ and $n=8 \pi f$. Note that the total energy of the crystal is extensive:

$$
E(N, M) \approx 2 N M E_{L}\left(\tilde{a} \equiv M a=\left(\frac{M^{3} V_{3}}{2 N}\right)^{\frac{1}{3}}\right) .
$$

In Fig. 8 we show the behavior of (51) for $\alpha_{s}=1$. The bcc configuration is bound for $\tilde{a}=M a \approx 5$, but the binding energy is very small: $E_{L} / M \approx-0.004$.

\section{B. Disordered crystal pressure}

The pressure for a disordered crystal follows from the corresponding partition function

$$
\Omega_{\mathrm{bcc}}(\tilde{a})=\sum_{N} \frac{\left(V_{3} 2 \pi \tilde{f}\right)^{4 N}}{(N !)^{4}} e^{-\frac{2 N M}{T} E_{L}(\tilde{a})}
$$

where we used the quantum and dressed fugacity $2 \pi \tilde{f}=$ $2 \pi f \sqrt{4 \nu \bar{\nu}}$ from (20). In the large $\mathrm{N}$ limit, the pressure $\mathcal{P}=\ln \Omega_{\mathrm{bcc}} / V_{3}$ can be cast in the form 


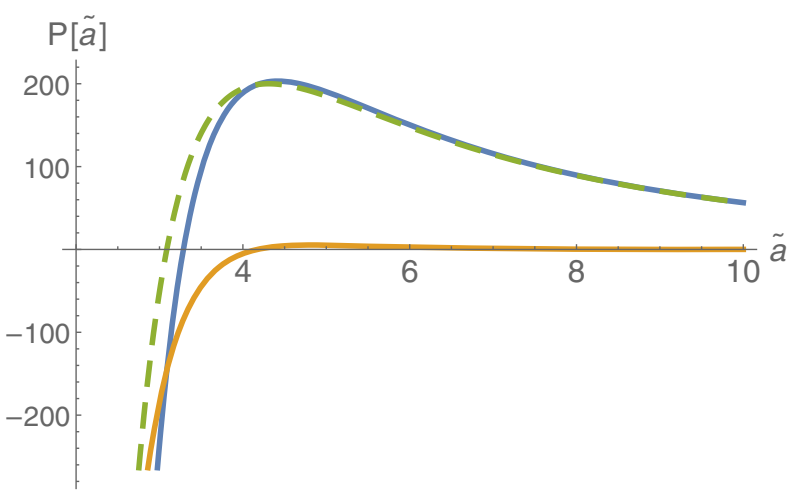

FIG. 9. Pressure (54) versus $\tilde{a}$ for $m=20$ and $\nu=\frac{1}{2}$ (upper solid curve). The separate contributions from the crystal [first term in (54)] are shown as the lower solid curve, and the entropy of the resummed trees [second term in (54)] is shown as the dashed curve.

$$
\frac{\mathcal{P}(\tilde{a})}{T^{3}} \approx-\frac{m^{4}}{\tilde{a}^{3}} E_{L}(\tilde{a})+\frac{2 m^{3}}{\tilde{a}^{3}}\left(1+\ln \left(\frac{\tilde{a}^{3} \sqrt{\nu \bar{\nu}}}{2 m}\right)\right)
$$

with $m=M / T$ (the ratio of the screening mass to the temperature). The first contribution in (54) is the crystal energy, and the second contribution is the entropy of the competing trees at large density as discussed in Sec. IVA. For large $m$ (very low temperature) the pressure is dominated by the crystal contribution, while for small $\mathrm{m}$ (intermediate temperature) the pressure is dominated by the entropy of the trees. In Fig. 9 we show the behavior of the pressure $\mathcal{P}(\tilde{a})$ versus $\tilde{a}$ for $m=20$ for the centersymmetric case with $\nu=\frac{1}{2}$ in the upper solid curve, while the crystal contribution is shown as the lower solid curve, and the tree contribution as the dashed curve. The pressure is maximum at

$$
\begin{aligned}
\frac{\mathcal{P}_{\max }}{T^{3}}= & \frac{m^{2}}{2} \sqrt{4 \nu \bar{\nu}} e^{-\frac{m}{2}\left(E_{L}\left(\tilde{a}_{\star}\right)-\tilde{a}_{\star}{ }^{3} E_{L}^{\prime}\left(\tilde{a}_{\star}\right)\right)} \\
& \times\left(1-\frac{m \tilde{a}_{\star}^{3}}{2} E_{L}^{\prime}\left(\tilde{a}_{\star}\right)\right)
\end{aligned}
$$

with $\tilde{a}_{\star}$ the solution to the transcendental equation

$$
\frac{\tilde{a}_{\star}^{3} \sqrt{\nu \bar{\nu}}}{2 m}=e^{\frac{m}{2}\left(E_{L}\left(\tilde{a}_{\star}\right)-\tilde{a}_{\star}^{3} E_{L}^{\prime}\left(\tilde{a}_{\star}\right)\right)} .
$$

If we were to assume $E_{L}$ fixed at the crystal minimum and constant as in Fig. 8, i.e., $E_{\mathrm{L} \min } \approx-0.004$, then (55) simplifies

$$
\frac{\mathcal{P}_{\max }}{T^{3}} \rightarrow \frac{m^{2}}{2} \sqrt{4 \nu \bar{\nu}} e^{-\frac{m}{2} E_{L \min }}
$$

which is seen to interpolate between the resummed tree contribution (29) at small $m$ (intermediate temperature) and the crystal at large $m$ (very low temperature). Due to the small binding energy of the crystal shown in Fig. 8, the crystal contribution takes over only when $\frac{m}{2}$ is large or very high density (very low temperature). This is confirmed numerically. Note that in both (54) and (57) the ratio $\frac{m}{2}$ plays the role of the Coulomb factor. It is rather large with $\frac{m}{2}=500$ for the onset of the crystal.

\section{CONCLUSIONS}

We have provided a many-body analysis of the instanton-dyon liquid model in the center-symmetric phase. The starting point of the analysis was a linearization of the moduli interactions between like instanton dyons $D D$ and anti-instanton dyons $\bar{D} \bar{D}$, followed by a cluster expansion. This reorganization of the many-body physics was shown to be captured exactly by a three-dimensional effective theory between charged particles. A semiclassical treatment of this effective theory amounts to resumming the tree contributions in the form of effective fugacities, while the one-loop correction amounts to resumming all ring or chain diagrams with effective fugacities. The tree or chain contributions are found to yield a center-symmetric phase even at a finite vacuum angle. They are dominant in the range $1 \leq \frac{m}{2} \leq 10$.

At very low temperature or large fugacities, an even larger class of diagrams needs to be resummed. In this vein we have carried the HNC resummation, as is commonly used for dense and charged liquids, and used it to estimate the pair correlation function around the DH approximation in the dense instanton-dyon liquid. The very low temperature phase is argued to be a melted bcc crystal with strong local topological and magnetic correlations. A simple description of the thermodynamics of an ensemble composed of trees and bcc crystals shows that the treelike contributions are dominant for most temperatures, with the exception of the very low temperature regime where the crystal arrangement is more favorable owing to its very small binding.

In sum, our analysis supports the bionlike description in [7] at low densities, and confirms the existence of a strongly screened phase at higher densities in line with our earlier investigations [1]. However, in contrast to [1], the present analysis allows for a dynamical determination of the confining holonomy in the dense regime to be $\frac{1}{2}$ as assumed in [1]. The resummation allowed for an explicit derivation of the critical temperature as a function of the vacuum angle, which for small angles is in agreement with currently reported lattice results [14]. The (disordered) crystal arrangement suggested in the very dense regime close to the vacuum state at zero temperature implies dual roles for instantons and monopoles as further detailed in [18].

\section{ACKNOWLEDGMENTS}

This work was supported in part by the U.S. Department of Energy under Contract No. DE-FG-88ER40388. 


\section{APPENDIX: MOLECULAR DYNAMICS}

Equation (1) describes a four-species ensemble of charged particles in three spatial dimensions. For fixed fugacity, the statistical ensemble described by (1) can be recovered from ensembles of classically evolved electrically and magnetically charged particles in three dimensions by sampling over random initial conditions. All the particles carry equal (dimensionless) mass $m_{D}=f^{\frac{2}{3}} / 2 \pi T^{2}$ and move classically following the Newtonian paths fixed by

$$
\begin{aligned}
& m_{D} \ddot{x}_{n}=-\frac{\partial}{\partial x_{n}} \sum_{[i, \bar{i}]}\left(V\left(x_{i}-y_{\bar{i}}\right)-\ln \operatorname{det} G\left[x_{i}\right]\right) \\
& m_{D} \ddot{y}_{\bar{n}}=-\frac{\partial}{\partial y_{\bar{n}}} \sum_{[i, \bar{i}]}\left(V\left(x_{i}-y_{\bar{i}}\right)-\ln \operatorname{det} G\left[y_{\bar{i}}\right]\right) .
\end{aligned}
$$

The first contribution is the Coulomb force stemming from the streamline potential, while the second contribution is the Coulomb force following from the moduli. The latter is of the form $\operatorname{Tr}\left(G^{-1} \partial_{n} G\right)$. It requires inverting $G$ at each time step, which may prove numerically costly for molecular dynamics (MD) simulations. It also requires that $\operatorname{det} G \neq 0$ for the inversion to be valid. For this the role of the initial conditions is important [1].
To remedy some of these shortcomings, we recall that a linearization of the effects induced by the moduli interactions amounts to nonlinear Debye-Huckel interactions between the pair $D, \bar{D}$ as captured by (10), leading to simpler MD equations

$$
\left(\begin{array}{l}
\mathbf{m}_{D} \ddot{x}_{D} \\
\mathbf{m}_{\bar{D}} \ddot{x}_{\bar{D}}
\end{array}\right)=\left(\begin{array}{c}
-\frac{\partial}{\partial x_{D}} \\
-\frac{\partial}{\partial x_{\bar{D}}}
\end{array}\right) \sum_{i=1,2 ; \underline{D} \underline{\bar{D}}} V_{i}\left(x_{\underline{D}}-x_{\overline{\bar{D}}} ; \theta\right) .
$$

Here the mass $\mathbf{m}_{D}$ is for the pair $D=L, M$ and $\mathbf{m}_{\bar{D}}=\mathbf{m}_{D}^{\dagger}$ for the pair $\bar{D}=\bar{L}, \bar{M}$ at finite vacuum angle

$$
\mathbf{m}_{D} \equiv\left(m_{L}, m_{M}\right)=m_{D} e^{\frac{i \theta}{3}}\left(\nu^{\frac{2}{3}}, \bar{\nu}^{\frac{2}{3}}\right) .
$$

The potentials in (A2) generalize (7) to finite vacuum angle

$$
\begin{aligned}
V_{1}(r ; \theta) & =-V_{2}(r ; \theta) \\
& =-\frac{C_{D}}{2 \alpha_{s} r}\left(e^{-M r e^{i \theta / 4}+i \theta}+e^{-M r e^{-i \theta / 4}-i \theta}\right) .
\end{aligned}
$$

Note that the MD analysis of (A2) for $\theta \neq 0$ is more challenging as it generates complex trajectories.
[1] Y. Liu, E. Shuryak, and I. Zahed, Phys. Rev. D 92, 085006 (2015); 92, 085007 (2015); 94, 105011 (2016); 94, 105012 (2016).

[2] T. C. Kraan and P. van Baal, Nucl. Phys. B533, 627 (1998); Phys. Lett. B 435, 389 (1998); K. M. Lee and C. Lu, Phys. Rev. D 58, 025011 (1998).

[3] D. Diakonov and V. Petrov, Phys. Rev. D 76, 056001 (2007); D. Diakonov and V. Petrov, AIP Conf. Proc. 1343, 69 (2011); D. Diakonov, arXiv:1012.2296.

[4] D. Diakonov, N. Gromov, V. Petrov, and S. Slizovskiy, Phys. Rev. D 70, 036003 (2004).

[5] R. Larsen and E. Shuryak, arXiv:1408.6563.

[6] A. R. Zhitnitsky, arXiv:hep-ph/0601057; S. Jaimungal and A. R. Zhitnitsky, arXiv:hep-ph/9905540; A. Parnachev and A. R. Zhitnitsky, Phys. Rev. D 78, 125002 (2008); A. R. Zhitnitsky, Nucl. Phys. A921, 1 (2014).

[7] M. Unsal and L. G. Yaffe, Phys. Rev. D 78, 065035 (2008); M. Unsal, Phys. Rev. D 80, 065001 (2009); E. Poppitz, T. Schafer, and M. Unsal, J. High Energy Phys. 10 (2012) 115; E. Poppitz and M. Unsal, J. High Energy Phys. 07 (2011) 082; E. Poppitz, T. Schafer, and M. Unsal, J. High Energy Phys. 03 (2013) 087.
[8] E. Shuryak and T. Sulejmanpasic, Phys. Rev. D 86, 036001 (2012); Phys. Lett. B 726, 257 (2013).

[9] P. Faccioli and E. Shuryak, Phys. Rev. D 87, 074009 (2013).

[10] E. Poppitz and T. Sulejmanpasic, J. High Energy Phys. 09 (2013) 128.

[11] M. C. Chu, J. M. Grandy, S. Huang, and J. W. Negele, Phys. Rev. D 49, 6039 (1994).

[12] T. Schfer and E. V. Shuryak, Rev. Mod. Phys. 70, 323 (1998).

[13] D. Diakonov and V. Petrov, Phys. Rev. D 67, 105007 (2003).

[14] M. D'Elia and F. Negro, Phys. Rev. Lett. 109, 072001 (2012).

[15] R. Larsen and E. Shuryak, Nucl. Phys. A950, 110 (2016).

[16] H. Frisch and J. L. Lebowitz, The Equilibrium Theory of Classical Fluids (Benjamin, New York, 1964).

[17] M. Rho, S. J. Sin, and I. Zahed, Phys. Lett. B 689, 23 (2010); I. Zahed, arXiv:1010.5980; P. Sutcliffe, Mod. Phys. Lett. B 29, 1540051 (2015); V. Kaplunovsky, D. Melnikov, and J. Sonnenschein, Mod. Phys. Lett. B 29, 1540052 (2015).

[18] A. Ramamurti, E. Shuryak, and I. Zahed, Phys. Rev. D 97, 114028 (2018). 